\title{
MATHEMATICIANS IN NUCLEAR REACTOR THEORY
}

\author{
B. Davison \\ (received May 23, 1958)
}

Among new fields of application of mathematics that have arisen since the war one of the more important is the theory of nuclear reactors. The reader need hardly be reminded of the role that atomic energy is bound to play in our future. As an indication, however, of the amount of unclassified research that has to go and is going into the development of its peaceful applications it can be mentioned that there are now several scientific periodicals both in the U.S.A. and in Britain entirely devoted to problems appertaining to nuclear reactors. Much of this work, in particular most of the work regarding the diffusion and slowing down of neutrons, is entirely mathematical in nature.

Unlike the older branches of applied mathematics, where the concern was invariably with differential equations, in the studies of neutron diffusion and slowing down one deals with integral and integro-differential equations (while the differential equations, where they enter at all, enter merely as an approximation to the appropriate integro-differential ones). This offers a much greater scope for the ability and ingenuity of an ambitious mathematician, and provides a welcome relief from the monotony of differential equations.

The needs of the neutron diffusion theory provided one of the main stimuli in the search for and the development of effective computational methods for the solution of integro-differential equations. Among the older developments in this direction one may mention the extension of the variational method and of the Wiener-Hopf method to inhomogeneous integral equations. Many well-known mathematical physicists, including Feynman, Wilson, Wick and Bethe, to mention just a few, have contributed to the development of the neutron diffusion and slowing down theory; but, while many problems are solved, many more await solution and many establishments and industrial organizations concerned with atomic energy are on the look-out for capable and resourceful mathematicians to get their teeth in to the problems yet unsolved. This in particular applies to the A.E.C.L. establishment at Chalk River, Ontario.

Many mathematicians may enquire how much physics they have to know in order to pursue successfully work in neutron

Can. Math. Bull., Vol.2, no. 1, Jan. 1959. 
diffusion theory. The answer is - their success will depend on their knowledge and ability as mathematicians and their ability to think in physical terms, rather than on any formal knowledge of physics. In the neutron diffusion theory one studies a stochastic process - outcome of a large number of events, the probability distribution of outcomes of individual events being prescribed beforehand. Formal knowledge of physics and of quantum mechanics would be needed to predict the probability distribution of the outcomes of individual events, but for the purposes of neutron diffusion theory it is quite irrelevant whether our knowledge of outcomes of individual events comes from modern physical theories or from direct experimental measurements. In so far as various mathematical techniques are concerned, the two that have been most helpful in the past are the Fourier-Laplace transform method and the expansions in orthogonal polynomials. It is, of course, impossible to say whether these two techniques will retain their leading importance or be superseded by some other methods, though one would expect the role played hy numerical methods steadily to increase.

Indeed, in recent years, increasing use has been made of high-speed electronic digital computers in solving problems of neutron diffusion as well as in other branches of applied mathematics. Since all but the simplest physical and geometrical situations in neutron transport theory lead to equations whose analytical solutions are unknown, the numerical techniques developed for obtaining approximate solutions are of great importance. Considerable work has been done in this field in the past ten years.

Apart from the straightforward evaluation of successive terms in an expansion, two different approaches have been used. The first consists of replacing differential equations by finite difference equations, and integrals by sums, and then using iterative methods to obtain solutions to the accuracy required. The other approach is the Monte Carlo method, which produces a controlled mathematical experiment in which the statistical processes of the pbysical model are simulated by numerical calculations involving random numbers.

Both of these general methods are capable of many refinements that can increase greatly their efficiency through speeding their convergence. There is still a great deal of progress to be made along the se lines, requiring a combination of mathematical and physical insight, accompanied perhaps by exploratory studies using a high-speed computer.

Apart from these long-range problems, there is a need for mathematicians who can appreciate the problems of physicists, chemists, and engineers, and can evolve numerical methods for solving these problems using a computer. 
A mathematician embarking on neutron transport theory work will much benefit, of course, from a reasonable familiarity with the above methods as well as with the general theory of integral equations. This will normally be found at the Ph.D. or at least at the M.Sc., rather than at the B.Sc. level. Also it will normally be found in those specializing in analysis or in numerical methods, rather than in abstract algebra, or theory of numbers, say. At the same time a man's ability is, of course, much more important that his background.

Computation Centre

University of Toronto.

\section{RANDOM NOTES FROM THE SECRETARIAT}

Benjamin Franklin says "three moves are as bad as a fire". We have now accomplished two moves and hope we won't have the fire in which case our work will be more disorganized than it is now. As the work of the Congress has increased, we have been in need of more and more space. We have now, thanks to our colleague Henri Gaudefroy, Director of the Ecole Polytechnique, and the Ecole Polytechnique board, two very commodious rooms in their new eleven million dollar building. We also have an attractive room in the Chemistry Building at McGill, thanks to Herbert Tate, Professor Winkler (Chairman of the Chemistry Department), and the McGill administration and governors. While there is some inconvenience in having offices four miles apart, we.hope so to divide our work between the two foci of the Congress as to carry it on without more than the ordinary confusion.

Lee Ritcey (see Volume 1, No.2, page 144 and No. 3, page 216) began his work as associate executive secretary-treasurer of the Congress on September lst. Let me say first of all that the confusion mentioned above is due to our recent move and to myself, and in no way to Dr. Ritcey and Mrs. Baker, both of whom have done their best to keep things on an even keel. As money - and I am sorry to take a materialistic view here - is one of the foundation stones on which the success of the Congress is built, his first task has been to visit a great many companies, particularly in Montreal and Toronto, soliciting funds, finding new subscribers and obtaining increased subscriptions from old subscribers. I am glad to report that he has had a good deal of success under somewhat difficult circumstances.

The Ritceys have moved in and gone to work without fuss and feathers. We do wish, however, to show our appreciation of them and give them a formal welcome, and are planning a reception at the Cercle Universitaire on December 9. In addition to members 